\title{
Pseudoscorpionida (Arachnida) em galerias de colônias de Passalidae (Coleoptera, Insecta) em troncos caídos em floresta de terra firme da Amazônia, Brasil
}

\author{
Nair Otaviano AGUIAR ${ }^{1}$, Paulo Friedrich BÜHRNHEIM ${ }^{1 \dagger}$ \\ RESUMO \\ Em sete municípios do Amazonas, um de Rondônia e um de Roraima, foram examinadas 71 colônias de 24 espécies de Passalidae \\ (Coleoptera), pertencentes aos gêneros: Passalus Fabricius, 1792 (14 espécies); Paxillus Mac Leay, 1819 (três); Popilius Kaup, \\ 1871 (três); Spasalus Kaup, 1869 (uma); Verres Kaup, 1871 (uma); Veturius Kaup, 1871 (duas). Foram registradas doze espécies \\ de pseudoscorpiōes, incluindo nove gêneros e cinco famílias, listadas a seguir: Chernetidae - Americhernes aff. incertus Mahnert, \\ 1979, Cordylochernes scorpioides (Linnaeus 1758), Lustrochenes similis (Balzan 1892), L. aff. reimoseri Beier, 1932, L. intermedius \\ (Balzan 1892), Phymatochernes crassimanus Mahnert 1979; Chthoniidae - Pseudochthonius homodentatus Chamberlin, 1929; \\ Lechytiidae - Lechytia chthoniiformis (Balzan 1887); Tridenchthoniidae - Tridenchthonius mexicanus Chamberlin \& Chamberlin \\ 1945; Withiidae - Cacodemoniussp., Dolichowithius (D.) emigrans (Tullgren 1907), D. (D.) mediofasciatus Mahnert, 1979. Dentre \\ as espécies mais freqüentes ( $T$. mexicanus, $L$. intermedius e $L$. aff. reimoseri), ocorreram todos os estágios de desenvolvimento. \\ Foram coletadas de uma a três espécies de pseudoscorpióes em cada colônia individual de besouros passalídeos. T. mexicanus \\ foi a única espécie encontrada em todos os municípios, ocorrendo em 45 colônias de dezenove espécies de passalídeos, sendo a \\ maioria dos exemplares encontrado no subcórtex. L. intermedius foi a segunda espécie mais abundante, ocorrendo em colônias \\ de 11 espécies de Passalidae, a maioria também no subcórtex. L. aff reimoseri ocorreu em 13 colônias de Passalidae, sob a casca, \\ alburno e cerne. $L$. aff. reimoseri foi a única espécie coletada somente no cerne.
}

PaLAVRAS-CHAVE: Pseudoscorpiones, Arachnida, Coleoptera, Passalidae, Amazônia, tronco caído.

\section{Pseudoscorpionida (Arachnida) found in the galleries made by Passalidae (Coleoptera, Insecta) colonies inside fallen wood of the Amazonian terra firme forest, Brazil}

\section{ABSTRACT}

Seventy-one colonies from 24 species of Coleoptera, Passalidae, belonging respectively to the genera Passalus Fabricius, 1792 (14 species), Paxillus Mac Leay, 1819 (three species), Popilius Kaup, 1871 (three species), Spasalus Kaup, 1869 (one species), Verres Kaup, 1871 (one species) and Veturius Kaup, 1871 (two species), were examined from seven municipalities of Amazonas, one of Rondônia and one of Roraima states. Twelve species of psedoscorpions were found, belonging to nine genera and five families, as follows: Chernetidae - Americhernes aff. incertus Mahnert 1979, Cordylochernes scorpioides (Lin. 1758), Lustrochenes similis (Balzan 1892), L. aff. reimoseri Beier, 1932, L. intermedius (Balzan 1892), Phymatochernes crassimanus Mahnert 1979; Chthoniidae - Pseudochthonius homodentatus Chamberlin 1929; Lechytiidae - Lechytia chthoniiformis (Balzan 1887); Tridenchthoniidae - Tridenchthonius mexicanus Chamberlin \& Chamberlin 1945; Withiidae - Cacodemonius sp, Dolichowithius emigrans (Tullgren 1907), D. mediofasciatus Mahnert, 1979. Among the most common species, all the development stages of T. mexicanus, L. intermedius and L. aff. reimoseri were found. From one to three species of pseudoscorpions were collected in each colony of passalíd beetles. T. mexicanus, the only species found in all municipalities, was caught in 45 colonies of nineteen species of passalids, the majority of them occurring under bark. L. intermedius, the second most abundant species, was caught in colonies of 11 species of passalids, the majority of them also under bark. Lustrochenes aff. reimoseri was caught in colonies of 13 species of Passalidae under bark and in sapwood and heartwood and was the only species caught exclusively in heartwood.

KEYWORDS: Pseudoscorpiones, Arachnida, Coleoptera, Passalidae, Amazon, fallen wood.

\footnotetext{
1 Universidade Federal do Amazonas, Instituto de Ciências Biológicas, Av. Rodrigo Octávio, 3000, Coroado, Manaus, AM. 69077-000. E-mail: naguiar@ufam.edu.br

+ "In Memoriam Postuma"
} 


\section{INTRODUÇÃO}

As galerias que besouros passalídeos escavam em troncos caídos e em decomposiçáo oferecem um lugar de excelente abrigo para vários grupos de microartrópodes, além de fornecer recursos alimentares a esses animais, o que por sua vez, poderá também atrair pequenos predadores, como os pseudoscorpiôes. Alguns estudos demonstraram que é muito freqüente a dispersão de pseudoscorpiōes por forésia em passalídeos. Como exemplo, Reyes-Castillo e Hendrich (1975), citam espécies de pseudoscorpiôes em forésia com espécies de passalídeo, ocorrendo no México e Aguiar e Bührnheim (1992a; 1998b), registram várias espécies desses aracnídeos sendo transportadas por muitas espécies de passalídeos na Amazônia.

Os estudos que tratam da fauna de pseudoscorpióes em troncos caídos e em decomposição são raros. $\mathrm{Na}$ Amazônia, o que se conhece sobre a fauna de pseudoscorpiōes desse hábitat foi citado por Mahnert e Adis (1985), quando listam todas as espécies de Pseudoscorpiones, até então conhecidos na região, incluindo dados sobre seus hábitats, citam algumas espécies vivendo em troncos. Entretanto, os registros de pseudoscorpiôes colonizando galerias de besouros da família Passalidae são referidos somente por: Fonseca (1988), que assinalou a presença de uma espécie de pseudoscorpiōes (não identificada) dentre a "fauna acompanhante" dentro de galerias de duas espécies desses besouros. Aguiar e Bührnheim (1998a) citam quatro espécies desses aracnídeos ocorrendo dentro de galerias de passalídeos, na ilha de Maracá, Roraima. Mouzinho e Fonseca (1998) citam uma espécie de pseudoscorpião, Tridenchthonius mexicanus, em galeria de Passalus interruptus (Linnaeus1758), encontradas em um tronco, em terra firme, no município de Manacapuru (Amazonas).

Este trabalho teve como objetivo, além de ampliar os registros em várias localidades da Amazônia brasileira, dar a conhecer a fauna de pseudoscorpióes, que colonizam galerias de besouros passalídeos. Bem como, sua distribuição por camada do tronco (subcórtex, alburno e cerne), espécies mais freqüentes e estágios de desenvolvimento encontrados nas galerias, assim como espécies que convivem juntas em uma mesma colônia. Dessa forma, é possível relacionar as espécies foréticas com seus transportadores, no ambiente onde convivem. A identificação de outras espécies que aí podem viver também é feita, porém sem estabelecer conexão com o processo de forésia.

\section{MATERIAL E MÉTODOS}

Foram realizadas coletas em vinte e duas localidades na Amazônia brasileira: uma no estado de Rondônia (RO), uma em Roraima (RR) e vinte no estado do Amazonas (AM). A maioria das coletas foi realizada em localidades de municípios dentro do inter-flúvio Juruá-Purus, na Província Petrolífera de Urucu, administrada pela PETROBRÁS em Coari (alto rio Urucu), Juruá e Uarini e as demais nos municípios de Beruri, Anamã, Manacapuru e Presidente Figueiredo (Tabela 1 e Figura 1). Os dados referentes ao município de Coari, fizeram parte da Tese de Doutorado da primeira autora, em Entomologia/INPA/UFAM (Aguiar 2000).

Em todas as localidades estudadas, foram examinados desde troncos finos até grandes troncos de árvores caídos em decomposição e encontrados aleatoriamente dentro da mata. Os troncos eram abertos com ajuda de machadinha e motoserra. $\mathrm{O}$ número de troncos variou conforme a disponibilidade em cada localidade visitada. Foram registrados somente os troncos colonizados por Coleoptera, Passalidae, onde foi constatada a presença de pseudoscorpióes. Foram examinados 69 troncos, dois deles (coletados no município de Beruri - Amazonas) com duas colônias, o que somou 71 colônias desses besouros. Dessas colônias, de 62 foi registrada sua localização nas camadas do tronco, assim enumeradas: $1=$ subcórtex; 2 = entre subcórtex/alburno; 3 = alburno; 4 = entre alburno/cerne; 5 = cerne; 6 = sem informação.

Todos os exemplares referidos neste estudo encontram-se conservados via úmida (álcool etílico a 70\%) e depositados na CZPB/UFAM - Coleção Zoológica Prof. Paulo Bührnheim, da Universidade Federal do Amazonas/ Instituto de Ciências Biológicas (ICB). Os espécimes de Pseudoscorpiones foram determinados por N.O. Aguiar e os de Passalidae por P.F. Bührnheim.

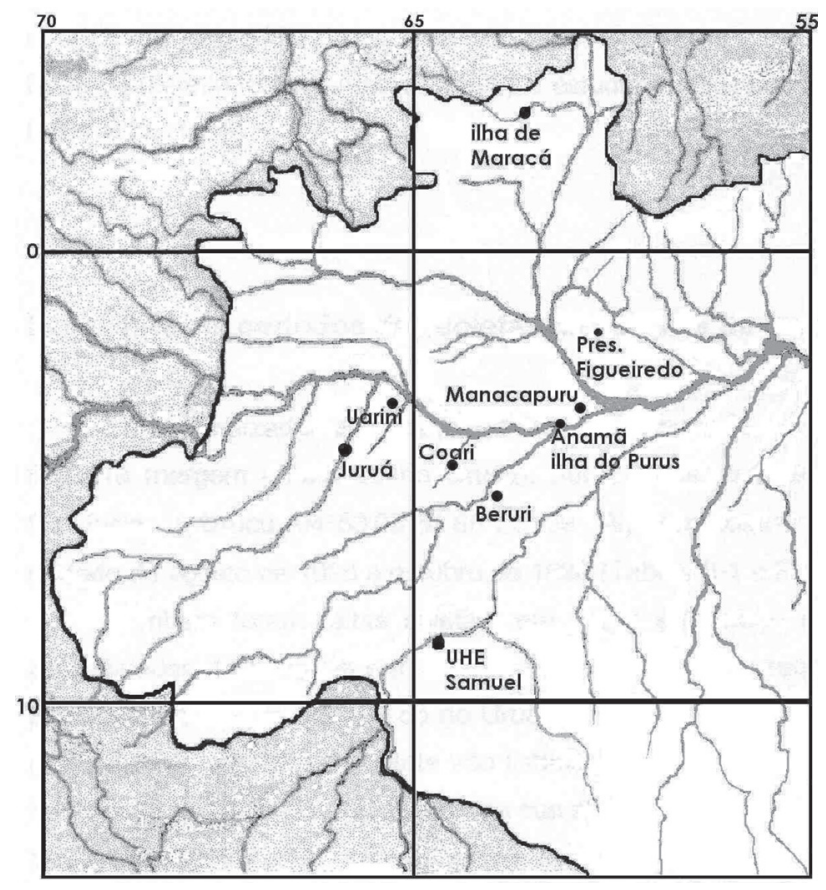

Figura 1 - Mapa indicando as localidades amostradas na Amazônia brasileira. 
Tabela 1- Localidades, na Amazônia, com seus respectivos períodos de coleta e total de colônias de Coleoptera-Passalidae examinadas em troncos caídos.

\begin{tabular}{|c|c|c|c|c|c|}
\hline UF & Município & Localidade & Coordenada & Período de coleta & No. de colônia \\
\hline \multirow{20}{*}{ AM } & Presidente Figueiredo & Rio Urubu & $02^{\circ} 10^{\prime} \mathrm{S} / 59^{\circ} 49^{\prime} \mathrm{W}$ & 06.ix.1983 & 9 \\
\hline & Presidente Figueiredo & UHE* do Pitinga & $00^{\circ} 51^{\prime} \mathrm{S} / 59^{\circ} 39^{\prime} \mathrm{W}$ & 23.ix.1991 & 3 \\
\hline & \multirow{12}{*}{ Coari } & Sumaúma & $04^{\circ} 49^{\prime} 23^{\prime \prime} \mathrm{S} / 64^{\circ} 55^{\prime} 02^{\prime \prime} \mathrm{W}$ & 01-10.iv.1991 & 1 \\
\hline & & LUC $^{*}-09$ & $04^{\circ} 51^{\prime} 56^{\prime \prime} \mathrm{S} / 65^{\circ} 00^{\prime} 56^{\prime \prime} \mathrm{W}$ & $\begin{array}{l}\text { 11-20.v.1991 e } \\
\text { 24.i-10.ii.1995 }\end{array}$ & 5 \\
\hline & & $R U C^{*}-29$ & $04^{\circ} 51^{\prime} 58^{\prime \prime} \mathrm{S} / 65^{\circ} 17^{\prime} 11^{\prime \prime W}$ & 01-10.ii.1992 & 3 \\
\hline & & $\mathrm{RUC}^{*}-30$ & $04^{\circ} 52^{\prime} 31^{\prime \prime} \mathrm{S} / 65^{\circ} 19^{\prime} 41^{\prime \prime} \mathrm{W}$ & 07-14.ix.1992 & 2 \\
\hline & & Angelim & $05^{\circ} 03^{\prime} 33^{\prime \prime} \mathrm{S} / 65^{\circ} 14^{\prime} 48^{\prime \prime} \mathrm{W}$ & 23.xi - 02.xii.1992 & 2 \\
\hline & & LUC*-18 & $04^{\circ} 53^{\prime} 54^{\prime \prime} \mathrm{S} / 65^{\circ} 11^{\prime} 58^{\prime \prime} \mathrm{W}$ & 19.ii - 01.iii.1993 & 4 \\
\hline & & SUC*-02 & $04^{\circ} 57^{\prime} 59^{\prime \prime S} / 65^{\circ} 19^{\prime} 39^{\prime \prime} \mathrm{W}$ & 14-24.v.1993 & 3 \\
\hline & & $\mathrm{IMT}^{*}-3$ & $04^{\circ} 50^{\prime} 01^{\prime \prime S} / 65^{\circ} 02^{\prime} 37^{\prime \prime} \mathrm{W}$ & 14-25.ix.1993 & 1 \\
\hline & & $R U C^{*}-27$ & $04^{\circ} 49^{\prime} 34^{\prime \prime S} / 65^{\circ} 15^{\prime} 38^{\prime \prime W}$ & 05-18.iii.1994 & 8 \\
\hline & & $R^{\prime} C^{*}-36$ & $04^{\circ} 55^{\prime} 55^{\prime \prime S} / 65^{\circ} 18^{\prime} 13^{\prime \prime W}$ & 25.ii - 10.iii.1995 & 2 \\
\hline & & $\mathrm{IMT}^{*}-1$ & $04^{\circ} 49^{\prime} 33^{\prime \prime} \mathrm{S} / 65^{\circ} 01^{\prime} 49^{\prime \prime} \mathrm{W}$ & 17-29.ix.1995 & 1 \\
\hline & & DUTO ** & $04^{\circ} 50^{\prime} 16^{\prime \prime} \mathrm{S} / 65^{\circ} 20^{\prime} 36^{\prime \prime} \mathrm{W}$ & 12-21.vi.1996 & 3 \\
\hline & Uarini & Rio Uarini & $03^{\circ} 02^{\prime} 57^{\prime \prime} \mathrm{S} / 65^{\circ} 41^{\prime} 42^{\prime \prime} \mathrm{W}$ & 25.vii.1995 & 1 \\
\hline & Juruá & Meneruazinho & $03^{\circ} 34^{\prime} 85^{\prime \prime} \mathrm{S} / 65^{\circ} 58^{\prime} 75^{\prime \prime} \mathrm{W}$ & 17.i.1996 & 2 \\
\hline & Beruri & \multicolumn{2}{|c|}{ Rio Purus, lago do Beruri } & 18.i.1987 & 2 \\
\hline & Anamã & \multicolumn{2}{|c|}{ Rio Solimões, ilha do Purus, Costa do Gabriel } & 12.i.1987 & 2 \\
\hline & Manacapuru & \multicolumn{2}{|c|}{ Estrada de Manacapuru/Novo Airão } & 06.ix.1987 & 2 \\
\hline & Manacapuru & \multicolumn{2}{|c|}{ Km 25 da Estrada Manuel Urbano } & 03.vii.1988 & 2 \\
\hline RO & Candeias & \multicolumn{2}{|c|}{ 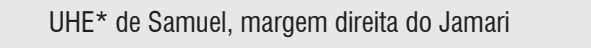 } & 04.xi.1986 & 2 \\
\hline \multirow[t]{2}{*}{$\mathrm{RR}$} & Amajari & \multicolumn{2}{|c|}{ Rio Uraricoera, ilha de Maracá } & 03.v.1987 & 11 \\
\hline & & & & Total de colônias & 71 \\
\hline
\end{tabular}

* RUC = Rio Urucu; LUC = Leste do rio Urucu; SUC = Sul do rio Urucu; IMT = Igarapé Marta; UHE = Usina Hidrelétrica.

** Duto Urucu/Porto Terminal, rio Tefé, única área à margem esquerda do rio Urucu.

\section{RESULTADOS}

Nos troncos examinados foram encontradas 24 espécies de Passalidae, distribuídas entre os gêneros: Passalus Fabricius, 1792 (com 14 espécies); Paxillus Mac Leay, 1819 (com três); Popilius Kaup, 1871 (com três); Spasalus Kaup, 1869 (uma); Verres Kaup, 1871 (uma); e Veturius Kaup, 1871 (duas). Das 62 colônias registradas sua localização no tronco, 29 foram encontradas no subcórtex, 2 entre o sub-cortex e alburno, 25 no alburno, 4 entre o alburno e cerne e 2 do cerne. Nas coletas realizadas em Coari, por duas ocasiōes, em um mesmo tronco, foi encontrado mais de uma espécie em uma só colônia, cujas galerias confundiam-se umas com as outras, sem ser possível distinguir os limites de cada uma. Nessa situação, coletaram-se juntos no subcórtex, Passalus interstitialis Eschcholtz, 1829, P. punctiger Lepelletier \& Serville, 1825 e Paxilus leachi Mac Leay, 1819 e em um tronco grande em galerias que iam do subcórtex ao alburno, Passalus rhodocanthopoides (Kuwert, 1891) junto com P. variiphylus (Kuwert 1891) (Tabela 2).

Levando em conta todas as colônias de passalídeos foram registradas doze espécies de pseudoscorpiōes, pertencentes 
Tabela 2 - Espécies de Passalidae habitantes de troncos caídos em cujas galerias ocorreram pseudoscorpiões, localidade em que ocorreram, camadas do tronco onde foram encontrados e número de colônias examinadas.

\begin{tabular}{|c|c|c|c|}
\hline Espécies de Passalidae & Procedências & Camada do tronco & No. de colônias \\
\hline •Passalus convexus Dalman, 1817 & AM (Coari e Pres. Figueiredo) & alburno & 3 \\
\hline Passalus elfriedae Luederwaldt, 1931 & AM (Pres. Figueiredo) & $?$ & 1 \\
\hline Passalus epiphanoides (Kuwert, 1891) & AM (Pres. Figueiredo) & $?$ & 2 \\
\hline -Passalus glaberrimus Eschcholtz, 1829 & AM (Coari) e RR & alburno & 4 \\
\hline •Passalus interruptus (Lin.,1758) & AM (Anamã, Beruri), R0 e RR & subcórtex e alburno & 8 \\
\hline •Passalus interstitialis Eschcholtz, 1829** & $\begin{array}{l}\text { AM (Beruri, Coari, Juruá e Uarini) } \\
\text { e RR }\end{array}$ & subcórtex & 14 \\
\hline •Passalus latifrons Percheron, 1841 & AM (Manacapuru, Pres. Figueiredo) & subcórtex e alburno & 3 \\
\hline Passalus aff nasutus Percheron, 1835 & AM (Juruá) & alburno & 1 \\
\hline Passalus punctiger Lep.\& Serv., 1825** & AM (Coari) & subcórtex & 2 \\
\hline Passalus rhodocanthopoides (Kuwert, 1891)* & AM (Coari) & subcórtex e alburno & 6 \\
\hline Passalus spinifer Percheron, 1841 & AM (Coari) & cerne & 1 \\
\hline Passalus unicornis Lep.\& Serv., 1825 & AM (Coari) & subcórtex & 2 \\
\hline Passalus aff unicornis Lep.\& Serv., 1825 & AM (Coari) & alburno & 2 \\
\hline Passalus variiphylus (Kuwert, 1891)* & $\begin{array}{l}\text { AM (Coari, Manacapuru, Pres. } \\
\text { Figeiredo) }\end{array}$ & alburno e cerne & 5 \\
\hline Paxillus camerani (Rosmini, 1902) & AM (Coari) & subcórtex & 2 \\
\hline Paxillus leachi Mac Leay, 1819** & AM (Coari) & subcórtex & 3 \\
\hline •Paxillus sp. & $\mathrm{RR}$ & subcórtex & 1 \\
\hline Popilius marginatus Percheron, 1835 & AM (Coari, Pres.Figeiredo) & alburno e cerne & 2 \\
\hline Popilius tetraphyllus (Eschcholtz, 1829) & AM (Coari) & alburno & 1 \\
\hline -Popilius $s p$ & AM (Coari) & alburno & 2 \\
\hline Spassalus crenatus (Mac Leay, 1819) & AM (Manacapuru) & alburno & 1 \\
\hline •Veres furcilabris (Eschcholtz, 1829) & AM (Coari, Pres.Figeiredo) e RR & alburno e cerne & 6 \\
\hline Veturius aff platyrhinus (Westwood, 1845) & AM (Coari) & alburno e cerne & 1 \\
\hline Veturius assimilis (Weber, 1801) & AM (Coari) & alburno & 1 \\
\hline
\end{tabular}

$\left.{ }^{*}\right)\left({ }^{\star *}\right)$ em uma das colônias coletadas em Coari ocorreram juntos na mesma galeria.

- Espécies já referida na literatura sobre a presença de pseudoscorpião em suas galerias

a nove gêneros de cinco famílias, cujas mais freqüentes e abundantes foram: Tridenchthonius mexicanus, Lustrochernes intermedius e L. aff. reimoseri (Tabelas 3, 4 e 5).

Tridenchthonius mexicanus foi a única espécie que ocorreu em todos os municípios estudados e em colônias de dezenove espécies de passalídeos (Tabela 3). Desse pseudoscorpião foram coletados 204 exemplares, de todos os estágios de desenvolvimento (Tabela 4) e entre as 90 fêmeas encontradas 34 estavam grávidas. A média de indivíduos por colônia foi de 4,53 e o número de indivíduos em uma colônia variou de 1 a 23, sendo esta a variação mais elevado dentre as espécies de pseudoscorpióes. Das 71 colônias de passalídeos estudadas em 45, ou seja, $63,4 \%$ foram coletados T. mexicanus e encontrados tanto sozinhos como acompanhados de até três outras espécies de pseudoscorpião, principalmente de L. intermedius, a segunda espécie mais abundante. A maioria das colônias habitadas por T. mexicanus, ou seja, 23 (51,1\%) encontravam-se no subcórtex e decresceu em direção ao cerne do tronco (Tabela 5).
Lustrochernes intermedius ocorreu em colônias de 11 espécies de Passalidae (Tabela 3), onde foram coletados 69 espécimes, ocorrendo todos os estágios de desenvolvimento (Tabela 4) e das 21 fêmeas ocorridas 3 portavam saco ovígero. Foram encontrados habitando 19 colônias das 71 examinadas, a maioria, ou seja, 15 (78,9\%) dessas colônias ocorreram no subcórtex (Tabela 5) e a variação do número de indivíduos em uma colônia foi entre 1 e 5 .

De Lustrochernes aff. reimoseri foram coletados 41 exemplares em colônias de 13 espécies de Passalidae (Tabelas 3 e 4). Ocorreram em 20 das 71 colônias examinadas e distribuídas desde o subcórtex ao cerne, cuja maioria, ou seja, 13 dessas colônias estavam localizadas entre o alburno e o cerne (Tabela 5). Esta foi à única espécie coletada exclusivamente no cerne, em colônias de Passalus spinifer e Verres furcilabris. A variação do número de indivíduos em uma colônia foi entre 1 e 4 . 
Tabela 3 - Pseudoscopionida em colônias de Passalidae coletados em várias localidades da Amazônia brasileira. AM (1 = Anamã; $2=$ Beruri; $3=$ Coari; $4=$ Juruá; 5 =Manacapuru 6 = Presidente Figueiredo; 7 = Uarini). R0 (8 = Usina Hidrelétrica de Samuel). RR (9 = ilha de Maracá, citadas em Aguiar e Bührnheim 1998a)

\begin{tabular}{|c|c|c|c|c|c|c|c|c|c|}
\hline Espécies de Pseudoscorpiones & & ocal & lidac & es & de 0 & cor & ênc & & \\
\hline Espécies de Passalidae & 1 & 2 & 3 & 4 & 5 & 6 & 7 & 8 & 9 \\
\hline CHERNETIDAE & & & & & & & & & \\
\hline $\begin{array}{l}\text { Amerchernes aff incertus } \\
\text { Mahnert,1979 }\end{array}$ & & & & & & & & & \\
\hline Passalus convexus* & & & & & & $x$ & & & \\
\hline Passalus interruptus & $x$ & & & & & & & & \\
\hline Passalus variiphylus* & & & $X$ & & & $x$ & & & \\
\hline Passalus rhodocanthopoides & & & $x$ & & & & & & \\
\hline Veres furcilabris & & & & & & $x$ & & & \\
\hline Cordylochernes scorpioides (Lin.,175 & & & & & & & & & \\
\hline Passalus rhodocanthopoides & & & $x$ & & & & & & \\
\hline Passalus interruptus & & $X$ & & & & & & & \\
\hline Paxillus leachi & & & $X$ & & & & & & \\
\hline Lustrochenes similis (Balzan,1892) & & & & & & & & & \\
\hline Passalus interstitialis & & & $X$ & & & & & & \\
\hline Lustrochernes aff reimoseri Beier, $19 ?$ & & & & & & & & & \\
\hline Passalus aff nasutus* & & & & $x$ & & & & & \\
\hline Passalus convexus* & & & & & & & & & $x$ \\
\hline Passalus epiphanoides & & & $\mathrm{x}$ & & & & & & \\
\hline Passalus glaberrimus* & & & $x$ & & & & & & \\
\hline Passalus interstitialis* & & & $x$ & & & & & & \\
\hline Passalus latifrons* & & & $x$ & & & $x$ & & & \\
\hline Passalus rhodocanthopoides* & & & $x$ & & & & & & \\
\hline Passalus spinifer* & & & $x$ & & & & & & \\
\hline Passalus variiphylus* & & & $x$ & & & & & & \\
\hline Popilius marginatus* & & & $x$ & & & & & & \\
\hline Popilius sp. & & & $x$ & & & & & & \\
\hline Verres furcilabris* & & & $x$ & & & & & & \\
\hline Veturius aff platyrhinus* & & & $x$ & & & & & & \\
\hline $\begin{array}{l}\text { Lustrochernes intermedius } \\
\text { (Balzan,1892) }\end{array}$ & & & & & & & & & \\
\hline Passalus aff unicornis & & & $X$ & & & & & & \\
\hline Passalus interruptus* & & $x$ & & & & & & & $x$ \\
\hline Passalus interstitialis* & & $x$ & $x$ & & & & & & $x$ \\
\hline Passalus latifrons & & & & & $x$ & & & & \\
\hline Passalus punctiger & & & $x$ & & & & & & \\
\hline Passalus rhodocanthopoides & & & $x$ & & & & & & \\
\hline Paxillus camerani & & & $x$ & & & & & & \\
\hline Paxillus leachi* & & & $x$ & & & & & & \\
\hline Paxillus sp. & & & & & & & & & $x$ \\
\hline Popilus marginatus & & & & & & $x$ & & & \\
\hline Verres furcilabris & & & & & & & & & $x$ \\
\hline $\begin{array}{l}\text { Phymatochernes crassimanus } \\
\text { Mahnert, } 1979\end{array}$ & & & & & & & & & \\
\hline
\end{tabular}

\begin{tabular}{llllllllll}
\hline Espécies de Pseudoscorpiones & \multicolumn{1}{c}{ Localidades de ocorrência } \\
\hline Espécies de Passalidae & 1 & 2 & 3 & 4 & 5 & 6 & 7 & 8 & 9 \\
\hline \multicolumn{1}{c}{ Passalus interstitialis } & $\mathrm{X}$ & & & & & & & \\
Passalus rhodocanthopoides & \multicolumn{1}{c}{$\mathrm{X}$} & & & & & \\
\hline
\end{tabular}

\section{CHTHONIIDAE}

Pseudochthonius homodentatus

Chamberlin, 1929

Passalus elfriedae

$X$

Passalus variiphylus

$x$

LECHYTIIDAE

Lechytia chthoniiformis (Balzan,1887)

Passalus interstitialis

Verres furcilabris

TRIDENCHTHONIIDAE

Tridenchthonius mexicanus Chamb.\&

Chamb., 1945

$\begin{array}{lll}\text { Passalus aff unicornis } & \mathrm{X} & \\ \text { Passalus convexus }^{*} & \mathrm{X} & \mathrm{X} \\ \text { Passalus epiphanoides } & & \mathrm{X}\end{array}$

$\begin{array}{lllllllll}\text { Passalus glaberrimus } & & & \mathrm{X} & & & & \mathrm{X} \\ \text { Passalus interruptus * }^{*} & \mathrm{X} & & & & & & \mathrm{X} & \mathrm{X} \\ \text { Passalus interstitialis }^{*} & & \mathrm{X} & \mathrm{X} & \mathrm{X} & & \mathrm{X} & \mathrm{X} \\ \text { Passalus latifrons * }^{*} & & & & & \mathrm{X} & & & \end{array}$

Passalus punctiger

Passalus rhodocanthopoides * $\quad \mathrm{X}$

Passalus unicornis* $\quad X$

Passalus variiphylus * $\quad \mathrm{X}$

Paxillus camerani $\quad \mathrm{X}$

Paxillus leachi $\quad X$

Paxillus $s p$.

Popilius tetraphyllus $\quad \mathrm{X}$

Popilius sp. $\quad \mathrm{X}$

Spassalus crenatus $\quad \mathrm{X}$

Verres furcilabris $\mathrm{X}$

Veturius assimilis $\mathrm{X}$

\section{WITHIIDAE}

Cacodemonius $s p$

Paxillus camerani

X

Dolichowithius (D.) emigrans

(Tullgren,1907)

Passalus interstitialis

$X$
$X$

Dolichowithius (D.) mediofasciatus

Mahnert,1979 
Tabela 4 - Número de pseudoscorpiões coletados em 71 colônias de Passalidae, construídas em troncos caídos. $M=$ machos; $F=$ fêmeas; T = tritoninfa; $\mathrm{D}=$ deutoninfa; $\mathrm{P}=$ protoninfa.

\begin{tabular}{|c|c|c|c|c|c|c|c|c|}
\hline \multirow{2}{*}{ Familias e espécies de Pseudoscorpiones } & \multicolumn{6}{|c|}{ Número de indivíduos } & \multirow{2}{*}{$\begin{array}{l}\text { Total de } \\
\text { colônias }\end{array}$} & \multirow{2}{*}{$\begin{array}{l}\text { Média de } \\
\text { Ind./colônia }\end{array}$} \\
\hline & M & $\mathrm{F}$ & $\mathrm{T}$ & $\mathrm{D}$ & $P$ & Total & & \\
\hline \multicolumn{9}{|l|}{ Chernetidae } \\
\hline Amerchernes aff incertus & 3 & 4 & 4 & 3 & 3 & 17 & 6 & 2,83 \\
\hline Cordylochernes scorpioides & 3 & & & & & 3 & 3 & 1,00 \\
\hline Lustrochenes similis & 2 & & 1 & 2 & & 5 & 1 & 5,00 \\
\hline Lustrochernes aff reimoseri & 10 & 21 & 5 & 2 & 3 & 41 & 23 & 1,78 \\
\hline Lustrochernes intermedius & 17 & 21 & 12 & 13 & 6 & 69 & 20 & 3,45 \\
\hline Phymatochernes crassimanus & & 2 & 1 & & & 3 & 2 & 1,50 \\
\hline \multicolumn{9}{|l|}{ Chthoniidae } \\
\hline Pseudochthonius homodentatus & 1 & 1 & 1 & & & 3 & 3 & 1,00 \\
\hline \multicolumn{9}{|l|}{ Lechytidae } \\
\hline Lechytia chthoniiformis & 1 & & 1 & & & 2 & 2 & 1,00 \\
\hline \multicolumn{9}{|l|}{ Tridenchthoniidae } \\
\hline Tridenchthonius mexicanus & 74 & 90 & 20 & 10 & 10 & 204 & 45 & 4,53 \\
\hline \multicolumn{9}{|l|}{ Withiidae } \\
\hline Cacodemonius sp & 5 & 6 & 2 & & & 13 & 1 & 13,00 \\
\hline Dolichowithius (D.) emigrans & & 1 & 2 & & & 3 & 1 & 3,00 \\
\hline Dolichowithius (D.) mediofasciatus & & & 1 & & & 1 & 1 & 1,00 \\
\hline TOTAL & 116 & 146 & 50 & 30 & 22 & 364 & * & * \\
\hline
\end{tabular}

* Foram coletadas mais de uma espécies de pseudoscorpião em uma colônia (Tabela 5).

\section{DISCUSSÃO}

Este é o primeiro estudo sobre a composição taxonômica da fauna de Pseudoscorpionida dentro de galerias, construídas em troncos caídos por besouros passalídeos. Trabalhos sobre a biologia e história natural de Passalidae que colonizam troncos caídos em decomposição e que registram a presença desses aracnídeos em suas galerias, foram publicados por: Fonseca (1988) que os refere em galerias de $P$. convexus e $P$. latifrons, porém sem citar as espécies ocorridas; Mouzinho e Fonseca (1998) coletaram T. mexicanus sob os élitros de $P$. interruptus recolhido de dentro de galeria em tronco; e Aguiar e Bührnheim (1998a) em estudo sobre pseudoscorpióes coletados em diferentes ambientes na ilha de Maracá, Roraima, registram quatro das dezessete espécies ocorridas ( $L$. aff reimoseri, $L$. intermedius, $L$. chthoniiformis e $T$. mexicanus constam na Tabela 3), em galerias de oito espécies de Passalidae (P. convexus, $P$. latifrons, $P$. interruptus, $P$. interstitialis, $P$. glaberrimus, V. furcilabris, Paxillus sp e Popilius sp). Portanto, considerando as 24 espécies de passalídeos encontradas, a presença de pseudoscorpióes como fauna acompanhante em galerias foi registrada pela primeira vez para 16 delas (Tabela 2).
Na literatura, há registros dos hábitas ou prováveis hábitas para as espécies de pseudoscorpióes encontradas neste estudo e a maioria foi mencionada por Mahnert e Adis (1985) para troncos, como: P. crassimanus, T. mexicanus, D. (D.) emigrans, L. similis, L. intermedius e D. (D.) mediofasciatus, as duas últimas ainda foram assinaladas por esses autores em copa de árvore e coletadas pelo método "canopy fogging". Aguiar e Bührnheim (2003) referiram P. homodentatus e P. crassimanus também ocorrendo sobre a vegetaçáo do sub-bosque. Dessas espécies, a mais comum é $P$. homodentatus, assinalada na maioria dos estudos com fauna de solo em diversas localidades na Amazônia e em áreas inundáveis fazem migração vertical para abrigar-se na vegetação, durante o pulso de cheia e vazante dos rios (Adis e Mahnert 1993; Aguiar et al. 2006; Mahnert e Adis 1985; Morais 1985; Morais et al. 1997).

Entre as espécies de pseudoscorpióes ocorridas, duas são consideradas raras nas amostragens realizadas na Amazônia. Dolichowithius (D.) emigrans foi descrita a partir de exemplares encontrados em orquídeas coletadas em Manaus e levadas para o "Naturhistorischen Museum in Hamburg" e depois somente um exemplar fêmea foi assinalado por Mahnert 
Tabela 5 - Espécies de Pseudoscorpião distribuidas nas colônias de passalídeos por camada de tronco-caído. 1=subcórtex; 2=entre subcórtex/alburno; $3=$ alburno; $4=$ entre alburno/cerne; $5=$ cerne; $6=$ sem informação.

\begin{tabular}{|c|c|c|c|c|c|c|c|}
\hline \multirow{2}{*}{ Espécies de Pseudoscorpiones } & \multicolumn{6}{|c|}{ Camada do tronco } & \multirow{2}{*}{$\begin{array}{l}\text { Total de } \\
\text { colônias }\end{array}$} \\
\hline & 1 & 2 & 3 & 4 & 5 & 6 & \\
\hline \multicolumn{8}{|l|}{ Somente uma espécie numa colônia } \\
\hline Tridenchthonius mexicanus & 6 & 1 & 10 & 1 & & 1 & 19 \\
\hline Lustrochernes aff reimoseri & 2 & & 4 & 1 & 2 & 2 & 11 \\
\hline Lustrochernes intermedius & 2 & & & 1 & & 1 & 4 \\
\hline Cacodemonius sp & 1 & & & & & & 1 \\
\hline Lechytia chthoniiformis & & & 1 & & & & 1 \\
\hline Dolichowithius (D.) mediofasciatus & & & 1 & & & & 1 \\
\hline Pseudochthonius homodentatus & & & & 1 & & 1 & 2 \\
\hline Amerchernes aff. incertus & & & & & & 4 & 4 \\
\hline \multicolumn{8}{|l|}{ Duas espécies juntas numa mesma colônia } \\
\hline L. aff reimoseri $+T$. mexicanus & 2 & & 5 & & & & 7 \\
\hline L. intermedius + T.mexicanus* & $11^{*}$ & 1 & & & & & 12 \\
\hline L. intermedius + C. scorpioides* & 1 & & $1^{*}$ & & & & 2 \\
\hline A. aff. Incertus $+T$. mexicanus & & & 1 & & & & 1 \\
\hline \multicolumn{8}{|l|}{ Três espécies juntas numa mesma colônia } \\
\hline A. aff incertus + L.aff reimoseri + T. mexicanus & 1 & & & & & 1 & 2 \\
\hline L. aff reimoseri + P.crassimanus $+T$. mexicanus & 1 & & 1 & & & & 2 \\
\hline C. scorpioides $+L$. intermedius $+T$. mexicanus & 1 & & & & & & 1 \\
\hline \multicolumn{8}{|l|}{ Quatro espécies juntas numa mesma colônia } \\
\hline L. similis + T.mexicanus + L.chthoniifornis + D.(D.) emigrans & 1 & & & & & & 1 \\
\hline$($ TOTAL DE TRONCOS $=69)$ TOTAL DE COLÔNIAS & & & & & & & 71 \\
\hline
\end{tabular}

*Duas colônias num mesmo tronco.

(1979), também em Manaus. Já o material tipo de Lechytia chthoniiformis é procedente da bacia dos rios Paraná e Paraguai e foi registrado pela primeira vez na Amazônia por Aguiar e Bührnheim (1991), quando referiram 26 espécimes em forésia com o Cerambycidae, Stenodontes spinibarbis (Lin. 1758) (=Mallodon spinibarbis), em Roraima e na natureza foi mencionado um exemplar capturado em "photo-eclector" de tronco de árvore, em Manaus (Morais et al. 1997) e outro em galeria do passalídeo, V. furcilabris, em Roraima (Aguiar e Bührnheim 1998 a).

Dez das doze espécies de pseudoscorpióes ocorridas neste estudo já foram registradas em dispersão por forésia, na Amazônia, tendo como transportadores besouros passalídeos e mais duas outras famílias de coleópteros xilófagos (Cerambycidae e Platypodidae) (Aguiar e Bührnheim 1998b). Uma dessas espécies (T. mexicanus) também foi encontrada sobre o corpo de duas espécies de Passalidae: Passalus interstitialis e Ptichopus angulatus (Percheron, 1835) no México (Reyes-Castillo e Hendrichs 1975). Os registros dessa relação na Amazônia, mencionam $C$. scorpioides, L. similis e L. chthoniiformis, associados em forésia com Cerambycidae (Aguiar e Buhrnheim 1991; 1992b; 1998b) e D. (D.) mediofasciatus sendo transportado por Patypodidae (Aguiar et al. 1992; 1997). Cinco dessas espécies de pseudoscorpióes (A. aff incertus, L. intermedius, L. aff reimoseri, T. mexicanus e Cacodemonius sp) foram registradas em associação forética com Passalidae (Aguiar e Bührnhiem 1992a; 1998b). Apenas duas das espécies ( $P$. homodentatus e $D$. (D.) emigrans) não há registros na literatura referentes a forésia. Podemos inferir que as espécies de passalídeos em cujas galerias foram constatadas a presença de pseudoescorpiōes, mas sem registro sobre associação forética entre eles, podem atuar como possíveis transportadores desses pseudoescorpióes. 
A dispersão por forésia também foi registrada para $P$. crassimanus, visto que o material-tipo, procedente de Manaus, além de outros exemplares assinalados em Carauari e Coari (Amazonas) foram coletados em armadilhas de Malaise, considerando que aí chegaram sendo levados por um inseto voador (Aguiar e Bührnheim 1998b; Mahnert 1979).

Vale ressaltar que nesse estudo não foi coletado Parawithius (Victorwithius) gracilimanaus Mahnert, 1979, uma das espécies já registradas em forésia com cinco espécies de Passalidae ( $P$. elfriedae, $P$. interruptus, $P$. interstitialis, $P$. rhocanthopoides e $P$. unicornis) (op. cit.) e ainda foi mencionada por Mahnert e Adis (1985) ocorrendo em tronco de árvore e epífitas. Esse dado leva a crer que também possa viver dentro das galerias de seus transportadores, porém raras nesse habitat.

Diante das informaçóes dadas por Harvey (2009) e Mahnert e Adis (2002), sobre a distribuição geográfica de todas as espécies de pseudoscorpiōes que ocorrem na Amazônia, até então conhecidas, ampliamos a distribuição de algumas das espécies, para vários municípios em que ainda não foram registradas, como: A. affincertus - ocorrendo em Anamã (AM); C. scorpioides - em Beruri (AM); L. intermedius - em Beruri e Manacapuru (AM); P. homodentatus - em Manacapuru e Presidente Figueiredo (AM); T. mexicanus - em Anamã e Beruri (AM) e Candeias (RO); D. (D.) emigrans - em Coari (AM), e D. (D.) mediofasciatus - em Candeias (RO).

\section{AGRADECIMENTOS}

A PETROBRÁS - Exploração \& Produção Amazônia, pelo o apoio logístico e transporte durante as coletas em suas áreas de exploração no inter-flúvio Juruá-Purus.

\section{BIBLIOGRAFIA CITADA}

Adis, J.; Mahnert, V. 1985. On the natural history and ecology of Pseudoscorpiones (Arachnida) from an Amazonian black water inundation forest. Amazoniana, 9 (3):297-314.

Adis, J.; Mahnert V. 1993. Vertical distribuition and abundance of Pseudoscorpions (Arachnida) in the soil of two diferent Neotropical primary forests during the dry rainy seasons. Memoirs of the Queensland Museum, 33 (2):431-440.

Aguiar, N.O. 2000. Diversity and Natural History of Pseudoscorpions (Arachnida), in dryland forest, the upper river Urucu, Coari, Amazon. Tese de Doutorado, Instituto Nacional de Pesquisas da Amazônia/Fundação Universidade do Amazonas, Manaus, Amazonas. 225 pp (in Portuguese).

Aguiar, N.O.; Bührnheim, P.F. 1991. Phoretic Pseudoscorpiopines of Stenodontes spinibarbis (Lin., 1758) (Coleoptera) and redescription of Lechytia chthoniiformis (Balzan, 1890) (Pseudoscorpiones, Chthoniidae) of the Island of Maracá Roraima. Acta Amazonica, 21: 425-433 (in Portuguese).

Aguiar, N.O.; Bührnheim, P.F. 1992a. Pseudoscorpiones (Arachnida) in phoretic association with Passalidae (Insecta, Coleoptera) in
Amazon, Brazil. Amazoniana, 12(2): 187-205 (in Portuguese, with abstract in English).

Aguiar, N.O.; Bührnheim, P.F. 1992b. Phoretic pseudoscorpions of Cerambycidae (Coleoptera) and occurrence of Parachelifer lativittatus Chamberlim, 1932 (Pseudoscorpiones, Cheliferidae) in the Amazon. Boletim Museu Emilio Goeldi, série Zoologia, 8(2): 343-348 (in Portuguese, with abstract in English).

Aguiar, N.O.; Bührnheim, P.F. 1998a. Pseudoscorpions (Arachnida) of the Ilha de Maracá, p. 381-389. In: Milliken, W.; Ratter, J. A. (Eds). Maracá. The biodiversity and Environment of an Rainforest. John Wiley \& Sons Ltd. England.

Aguiar, N.O.; Bührnheim, P.F. 1998b. Phoretic Pseudoscorpions associated with flying Insects in Brazilian Amazonia. The Journal of Arachnology, 26: 452-459.

Aguiar, N.O.; Bührnheim, P.F. 2003. Pseudoscorpions (Arachnida) in the undergrowth vegetation in dryland forest in Coari, Amazon, Brazil. .Acta Amazonica, 33(3): 515-526 (in Portuguese, with abstract in English).

Aguiar, N.O.; Arruda, A.M.R.; Bührnheim, P.F. 1997. Pseudoscorpiones (Arachnida) in dispersion for phoresy on flying insects, in lumber industries of Manaus, Amazon. Revista da Universidade do Amazonas, Série Ciências Agrárias, 6(1): 79-89 (in Portuguese, with abstract in English).

Aguiar, N.O.; Gualbert, T.L.; Franklin, E. 2006. Medium-spatial scale pattern distribution of Pseudoscorpionida (Arachnida) in a gradient of topography (altitude and inclination) soil factors, and litter in a central Amazon forest reserve, Amazonas, Brazil. Brazilian Journal of Biology, 66(3):29-41.

Aguiar, N.O.; Silva, J.V.; Bührnheim, P.F. 1992. Dolichowithius mediofasciatus Mahnert, 1979 (Arachnida, Pseudoscorpiones, Withiidae) in phoresy with Platypodidae (Insecta, Coleoptera), in Amazon, Brazil. Amazoniana, 12(2):181-185 (in Portuguese, with abstract in English).

Fonseca, C.R.V. 1988. Contribution to the knowledge of the bionomy of Passalus convexus Dalman, 1817 and Passalus latifrons Percheron, 1841 (Coleoptera, Passalidae). Acta Amazonica, 18(12): 197-222 (in Portuguese).

Harvey, M.S. 2009. Pseudoscorpions of the world, version 1.2. Western Australian Museum, Perth (http://www.museum.wa.gov.au/ arachnids/pseudoscorpions/). Acesso em 15/1/2010.

Mahnert, V. 1979. Pseudoscorpions (Arachnida) from the Amazon region (Brazil). Revue suisse de Zoologie, 86 (3): 719-810 (in German, with abstract in English).

Mahnert, V.; Adis, J. 1985. On the occurrence and Habitat of Pseudoscorpiones (Arachnida) from Amazonian Forests of Brasil. Studies on neotropical Fauna and Environment, 20 (4): 211-215.

Mahnert, V.; Adis, J. 2002. Pseudoscorpiones, p. 367-380. In: Adis, J. (Ed). Amazonian Arachnida and Miriapoda. Pensoft Publishers. Sofia-Moscow.

Morais, J.W. 1985. Abundance and vertical distribution of soil arthropods in an unflooded primary forest. Dissertaçáo de Mestrado, Instituto Nacional de Pesquisas da Amazônia/ Fundação Universidade do Amazonas, Manaus, Amazonas. 92 pp (in Portuguese). 
Morais, J.W.; Adis, J.; Mahnert, V. 1997. Abundance and phenology of Pseudoscorpiones (Arachnida) from a mixedwater inundation forest in Central Amazonia, Brazil. Revue suisse de Zoologie 104(3): 475-483.

Mouzinho, J.R.C.; Fonseca, C.R.V. 1998. Contribution to the study of passalidofauna (Coleoptera, Scarabaeidea, Passalidae) in a dryland area of the Central Amazon. Acta Zoológica Mexicana (n.s), 73: 19-44 (in Portuguese, with abstract in English and Spanish).
Reyes-Castillo, P.; Hendrichs, J. 1975. Pseudoscorpiones associated with passalids. Acta Politécnica Mexicana, 16 (72):129-133 (in Spanish).

Recebido em 22/03/2010

Aceito em 09/07/2010 
